\title{
Aplicação do Modelo Multicritério Aditivo em uma Empresa de Doces em Pernambucano
}

\author{
Luan Emerson Soares de Lima - luanemerson8@gmail.com ${ }^{1}$ \\ Sóstenes Luiz Soares Lins - sostenes.lins@gmail.com²
}

\begin{abstract}
Resumo - Problemas relacionados à decisão permeiam nosso cotidiano, no entanto tão importante quanto à decisão é como realizá-la, na literatura existem as mais variadas técnicas para a realização de procedimentos decisórios, que podem ou não levar em conta a estrutura de preferência do decisor. Considerando a racionalidade limitada do decisor no processo decisório e a dificuldade de acesso de micro e pequenas empresas a tecnologias que favorecem a tomada de decisão, o presente trabalho aplica um Método de Agregação Aditivo Determinístico por meio do Modelo Multicritério proposto por Almeida (2013) a uma fábrica de doces de pequeno porte no Estado de Pernambuco. O método proposto é compensatório e integra as preferências do decisor. Ao longo do trabalho executa-se as etapas do modelo à medida que contextualiza-se o processo decisório.
\end{abstract}

Palavras-chave: Modelo Multicritério, Método de Agregação Aditivo Determinístico, Decisão.

\section{Application of the Additive Multicriteria Model in a Candy Company in Pernambucano}

\begin{abstract}
Problems related to the decision permeate our daily lives, however as important as the decision is how to make it, in the literature there are the most varied techniques for carrying out decision-making procedures, which may or not take the decision-maker's preference structure. Considering the limited rationality of the decision-maker in the decision-making process and the difficulty of access by micro and small companies to technologies that favor decision-making, this work applies a Deterministic Additive Aggregation Method through the Multicriteria Model proposed by Almeida (2013) to a small candy factory in the State of Pernambuco. The proposed method is compensatory and integrates the preferences of the decision maker. Throughout the work, the stages of the model are executed as the decision-making process is contextualized.
\end{abstract}

Keywords: Multicriteria Model, Deterministic Additive Aggregation Method, Decision. 


\section{Introdução}

Conforme Slack, Jones e Robert (20I6) a função central de qualquer empreendimento é a produção de bens e serviços que satisfaçam clientes, consumidores ou usuários.

As organizações requerem de seus sistemas produtivos uma eficiente capacidade de processamento, equilibrando a oferta de bens e serviços com elevado padrão de qualidade, com a diminuição de custos operacionais. A necessidade de eficiência e melhoramento constante afeta organizações de caráter público ou privado, com ou sem fins lucrativos, de pequeno ou grande porte.

Segundo Hillier e Lieberman (20I5) a eficiência organizacional está ligada diretamente a tomada de decisão da alocação dos recursos disponíveis nos empreendimentos.

Desde meados da Segunda Guerra Mundial, a Pesquisa Operacional (PO) oferece uma série de métodos ou técnicas matemáticas que dão suporte a tomada de decisão e solucionamento de problemas. De acordo com Goldbarg e Luna (2005) esses métodos ou técnicas fundamentamse de forma lógica, amparados em estruturas axiomáticas robustas, para representar da melhor maneira possível a realidade e garantir soluções satisfatórias.

As técnicas presentes na PO podem levar em conta a estrutura de preferência do decisor, como é o caso dos Métodos Multicritérios, bem como assumir o comportamento racional dele e tratar os problemas como claramente estruturados e definidos, nesse caso pode-se citar a Programação Matemática.

Quando tratamos de tomada de decisão, empresas de micro, pequeno e médio porte possuem pouco ou nenhum conhecimento sobre técnicas para alocação adequada de recursos.

Segundo dados do Sebrae até abril de 2017 no estado de Pernambuco existiam 373.535 mil negócios cadastrados como empresas de micro e pequeno porte, que representavam $26,1 \%$ do PIB do estado e empregavam $48,6 \%$ da força de trabalho (SEBRAE, 20I7).

Tendo em vista a significativa participação de empresas de micro, pequeno e médio porte na economia do estado, o presente trabalho utiliza o método de agregação aditivo determinístico para a ordenação dos produtos manufaturados por uma fábrica de doce, levando-se em conta as preferências do proprietário do negócio. Essa ordenação baseia-se nos objetivos estabelecidos a serem alcançados pela empresa e nos critérios de ordenação definidos e associados aos produtos ou alternativas.

A ordenação dos produtos evidencia quais itens deveriam ser priorizados na produção levando-se em conta a preferência dos decisores, o que garantiria uma melhor alocação de recursos e uma solução customizada, desta forma o trabalho utiliza a perspectiva construtivista na tomada de decisão.

O método de agregação aditivo determinístico será aplicado mediante o modelo proposto por Almeida (20I3) que envolve I2 etapas. Logo, além de executar a solução do problema de ordenação dos produtos preferíveis a serem produzidos em uma empresa de pequeno porte, $o$ trabalho também contextualiza as etapas propostas pelo autor. 


\section{Fundamentação Teórica}

\subsection{Referencial Retórico}

A tomada de decisão é uma atividade cotidiana e corriqueira, ela pode ocorrer nas mais amplas dimensões da vida humana. No entanto, devido à complexidade associada a determinadas escolhas faz-se necessário o uso de técnicas para suporte ao processo decisório.

Gigerenzer e Selten (2002) afirmam que em um mundo incerto e complexo, os homens tomam decisões sob restrições de conhecimento, recursos e tempo.

De acordo com Sharda, Delen e Turban (2019) a tomada de decisão envolve vários aspectos, dentre eles pode-se destacar a qualidade da informação utilizada e o grau de customização das soluções encontradas. Por customização entende-se a inclusão das preferências do decisor no processo decisório.

Quando o processo decisório requer informações estruturadas e nenhuma customização, pode-se utilizar técnicas clássicas da Programação Matemática (PM) para a resolução do problema, no entanto quando a informação é pouco estruturada ou semi-estruturada e a decisão requer a modelagem da preferência do decisor ,recomenda-se a utilização de Métodos Multicritérios para Apoio a Decisão ou Multi-Criteria Decision Analysis (MCDA).

$\mathrm{Na}$ Programação Matemática busca-se atender a uma função objetivo, já em Métodos Multicritérios almeja-se satisfazer o julgamento de valor e a subjetividade do decisor.

Apesar de tratarem a subjetividade do decisor, Métodos Multicritérios para Apoio a Decisão possuem em sua concepção uma estrutura axiomática bem definida e consolidada (Almeida, 2013).

Simon (I997) escreve que a mente humana não é capaz de levar em conta, em uma decisão, todos os aspectos de valor, conhecimento e comportamento que seriam relevantes para a escolha, logo esses aspectos impõem limites à racionalidade objetiva.

Segundo Pinto et al. (20I3) qualquer MCDA deve ter caráter científico e, ao mesmo tempo, subjetivo, trazendo consigo a capacidade de agregar, de maneira ampla, todos os aspectos considerados importantes para a escolha, inclusive os não quantitativos, objetivando possibilitar a transparência e a sistematização do processo de tomada de decisão.

Para Eisenhart e Zbaracky (1992), o processo decisório racional pode ser caracterizado pelos seguintes passos: Definição dos objetivos; Obtenção das informações; Desenvolvimento das alternativas e Escolha de uma alternativa. Logo destaca-se que, estes passos estão alinhados com o Modelo de Tomada de Decisão proposto por Almeida (20I3) e utilizado neste trabalho.

Conforme Almeida (20I3) problemas de decisão multicritério envolvem uma situação em que existem ao menos duas alternativas de ação para se escolher, a escolha será feita mediante o desejo de se alcançar múltiplos objetivos que quase sempre são conflitantes entre si, associado a esses objetivos existem critérios que representam as alternativas do problema avaliado. 
A esses objetivos estão associadas variáveis que os representam e permitem a avaliação de cada alternativa. Essas variáveis, por sua vez, recebem o nome de critérios, atributos ou dimensões (ALMEIDA, 20I3).

Santos, Lugo e Almeida (20I5) oferecem um exemplo do processo decisório da compra de um celular utilizando o método de agregação aditivo determinístico, onde os objetivos do decisor eram: Maximizar o tempo de duração da bateria; Maximizar o desempenho; Minimizar o peso do aparelho; Maximizar a memória interna e Minimizar o preço de compra. Como critérios associados aos objetivos tinham-se: Duração da bateria; Sistema operacional; Espessura; Memória interna e Preço. As alternativas de aparelho celular para escolha eram: Iphone ${ }_{5} \mathrm{~S}$; Samsung Galaxy S5; Iphone 6 plus; Motorola Moto Maxx e Iphone 6.

O método de agregação aditivo determinístico pode ser usado quando existe a natureza compensatória no processo de escolha do decisor. Apesar da utilização desse método para tomada de decisão ser comum, muitos erros e equívocos podem ocorrer durante sua aplicação. Almeida (20I3) em seu trabalho descreve o método e oferece importantes comentários sobre o assunto.

Wallenius et. al (2008) propõe a classificação dos MCDA conforme a natureza do conjunto de alternativas do problema. Logo, os MCDA podem ser definidos como possuidores de alternativas com características discretas ou contínuas.

Por outro lado, a literatura publicada ao longo dos anos consolida a divisão dos MCDA em três categorias (ROY, I996; PARDALOS et al., I995; VINCKE, I992; ALMEIDA, 20I3). Sendo elas:

a) Métodos de critério único de síntese: Analytic Hierarchy Process (AHP), SMARTS, SMARTER, Multi-Attribute Utility Theory (MAUT), Métodos de Agregação Aditivos Determinísticos e Modelo Aditivo com Veto;

b) Métodos de sobreclassificação (outranking): famílias ELECTRE, PROMETHEE;

c) Métodos interativos.

Ainda no campo teórico, Edwards, Miles Junior e Winterfeldt (2007) distinguem três perspectivas ligadas ao estudo da tomada de decisão: a Descritiva, a Normativa e a Prescritiva. A Descritiva trata de relatar como o decisor realiza escolha, a Normativa trata do estudo da estrutura axiomática necessária para agregar de maneira lógica as preferências do decisor, por fim, a perspectiva Prescritiva refere-se à aplicação da Normativa.

Bouyssou (2006) relata uma quarta perspectiva conhecida como Construtivista que corresponde à perspectiva Prescritiva de forma interativa, onde assume-se o paradigma da construção da aprendizagem do decisor, de modo a consolidar sua estrutura de preferência.

\subsection{Breve Estado da Arte}

Para um rápido levantamento do Estado da Arte presente na literatura científica em português sobre o assunto definiu-se o buscador na web conhecido como Google Scholar e as palavras-chave "Método de Agregação Aditivo", "Método Aditivo Determinístico" e "Método 
Aditivo". A partir da obtenção dos resultados de pesquisa foram selecionados apenas os trabalhos que fossem artigos científicos e estivessem fielmente ligados ao tema.

Utilizando-se as palavras-chave "Método de Agregação Aditivo", sem limitação de data de publicação, foram encontrados 37 resultados dos quais dois estavam relacionados ao assunto, a saber: Santos et al. (2017) e Evair et al. (2019).

Santos et al. (2017) aplicam o método aditivo com veto ao processo de priorização de fornecedores em uma empresa de confecção e Evair et al. (20I9), por sua vez, utilizam o modelo aditivo com veto para a problemática de escolha de um novo fornecedor no estudo de caso de uma empresa ideal.

As palavras-chaves "Método Aditivo Determinístico" ofereceram 7 resultados, desses resultados apenas 3 estavam relacionados ao tema: Evair et al. (2019) ; Turet e Daher (2016) e Santos, Lugo e Almeida (20I5), cabe salientar que o trabalho de Evair et al. (2019) já foi comentado anteriormente. Para estas palavras-chaves também não definiu-se limites mínimos ou máximos de data de publicação.

Santos, Lugo e Almeida (2015) utilizam o método aditivo determinístico para decidir que celular deveria ser comprado levando em conta características consideradas essenciais pelo decisor.

Turet e Daher (20I6) aplicam o método aditivo com veto para a elaboração de um plano de ação em um m-commerce.

Limitando-se a busca de publicações para aproximadamente os últimos 2 anos, período entre janeiro de 2018 e abril de 2020, e utilizando-se o termo "Método Aditivo", encontrou-se apenas o trabalho de Evair et al. (2019) na ferramenta.

A partir dos resultados das pesquisas acima percebeu-se o baixo número de publicações ligadas ao Método de Agregação Aditivo Determinístico, bem como destaca-se a adição de veto em quatro dos trabalhos analisados.

A adição de veto justifica-se por limitar o efeito compensatório intercritério, esse mecanismo é proposto por Almeida (2013) e estabelece a inclusão de uma penalização intracritério, com a especificação de limiares máximos e mínimos para os valores que os critérios possam assumir.

\subsection{Método de Agregação Aditivo Determinístico}

Conforme Almeida (20I3) para a aplicação do método de agregação aditivo determinístico considera-se uma situação de certeza na obtenção de um vetor $x$ de consequências para cada $a$ alternativa. Dado $n$ critérios, para cada alternativa têm-se a Equação I abaixo, que representa a avaliação intracritério do método, onde $v(a)$ representa o valor final de dada alternativa; $k_{j}$ representa a constante de escala ou taxa de substituição expressa pela escolha da consequência de um critério em detrimento a consequência de outro; e $v_{j}(x)$ é o valor do critério $j$ normalizado. 


$$
v(a)=\sum_{j=1}^{n} k_{j} v_{j}(x) \quad \text { Equação(1) }
$$

Cabe salientar que $k_{j}$ representa a constante de escala para cada critério $j$, veja a Equação 2:

$$
\sum_{j=1}^{n} k_{j}=1 \quad \text { Equação (2) }
$$

A alternativa que oferecer o maior valor para $v(a)$ será a melhor opção para o problema multicritério.

\subsection{Etapas do Modelo de Decisão Multicritério}

O Modelo de Decisão de Problemas Multicritério exemplificado abaixo e suas I2 etapas foram propostas por Almeida (2013).

\subsubsection{Etapa 1. Caracterização dos Decisores e Outros Atores}

Denomina-se decisor o indivíduo responsável pela escolha de determinada alternativa em um problema de decisão multiobjtivo, a essas escolhas estão associadas consequências, os impactos e desdobramentos dessas consequências, por sua vez, serão respondidas pelo decisor.

Entre os atores presentes em um problema multicritério, ainda pode-se destacar o analista, o especialista e os stakeholders. Por analista entende-se o individuo responsável pela confecção do modelo de decisão multicritério e agregação das preferências do decisor. Ao especialista cabe o fornecimento de informações especializadas e conhecimentos sensíveis ao modelo. Os stakeholders são os atores que sofreram as consequências tomadas pelo decisor e por isso influenciam no processo de tomada de decisão.

\subsubsection{Etapa 2. Identificação dos Objetivos}

Nessa etapa são definidos os objetivos fundamentais e os objetivos - meio almejados pelo decisor no processo decisório.

\subsubsection{Etapa 3. Estabelecimento dos Critérios}

O estabelecimento dos critérios está diretamente ligado aos objetivos do processo decisório. Conforme Keeney (1992) eles irão medir o grau de desempenho dos objetivos para cada alternativa de escolha.

Os critérios também podem ser classificados conforme a escala de avaliação a que pertencem.

\subsubsection{Etapa 4. Estabelecimento do Espaço de Ações e Problemática}

Nessa etapa são geradas as alternativas de escolha do processo decisório e a determinação da problemática, ou seja, como o resultado pretendido será determinado, se por meio de uma escolha, por uma ordenação de alternativas ou mesmo classificação, dentre outras possibilidades. 


\subsubsection{Etapa 5. Identificação de Fatores Não Controlados}

Fatores não controlados são aspectos não explícitos ou sujeitos a incertezas e risco. Eles precisam ser identificados, pois podem influenciar no processo de tomada de decisão.

\subsubsection{Etapa 6. Modelagem de Preferências}

Nessa etapa estuda-se se as características de preferência do decisor são compensatórias ou não. Em problemas compensatórios, o maior desempenho da consequência desejada por determinado critérios pode ser compensada pelo menor desempenho de outro critério, no entanto, no caso de estruturas não compensatórias, esse fenômeno não ocorre.

\subsubsection{Etapa 7. Avaliação Intracritério}

Diferentes métodos multicritério possuem as mais distintas formas de avaliação intracritério. No entanto, para o Método de Agregação Aditivo Determinístico e outros métodos compensatórios, conforme Almeida (2013), a avaliação intracritério consiste na normalização dos valores dos critérios para cada alternativa presente. Ressalta-se a importância da utilização do procedimento de normalização (Equação 4) para transformar os dados numa escala de 0 a I, na qual o o, para este caso, significa apenas o valor mínimo, dessa forma, $v^{\prime} j$ (ai) representa o valor final normalizado, vj(ai) o valor a ser normalizado, Min vj(ai) o mínimo valor que o critério pode assumir quando cada alternativa é observada e Max vj(ai) o máximo valor para o critério.

$$
v^{\prime} j(a i)=\frac{[v j(a i)-M i n v j(a i)]}{[\operatorname{Max} v j(a i)-M i n v j(a i)]} \quad(\text { Equação } 4)
$$

Procedimentos onde deseja-se minimizar os objetivos relacionados aos critérios utilizam a Equação 5 .

$$
v^{\prime} j(a i)=\frac{1}{v j(a j)} \quad(\text { Equação 5) }
$$

\subsubsection{Etapa 8. Avaliação Intercritério}

Em métodos compensatórios o procedimento para elicitação da constante de escala ou taxa de substituição $k_{j}$ ocorre via trade-off por meio da troca da preferência da consequência associada a um critério pela consequência associada a outro. Já em métodos não compensatórios essa constante revela o grau de importância dos critérios e não é uma compensação entre consequências. Para todo efeito, essa constante pode ser chamada de parâmetro.

De acordo com Almeida (20I3) a elicitação ocorre em seis passos para métodos compensatórios, conforme pode-se verificar abaixo. O objetivo final dessas etapas é encontrar a taxa de substituição quando comparadas as consequências par a par para cada critério:

Etapa I : Avaliação intracritério

Nesta etapa obtêm-se o valor de consequência $v_{j}\left(x_{j}\right)$ para cada critério j.

Etapa 2: Ordenação dos critérios

Ordenação da valor das consequências da melhor para a pior, por exemplo $k_{2}>k_{I}$, dentro 
de um conjunto de $k_{n}$ consequências, ou seja, a consequência do critério 2 e preferível a do critério I.

Etapa 3: Explorando mais o espaço de conseqüências

Analisam-se as relações de preferência, buscando-se inequações entre os valores das consequências, para que o decisor possa pensar mais no espaço de consequências;

Etapa 4: Obtenção da relação entre as constantes de escala

Atribui-se valores para as consequências ordenadas, da mais preferível a menos preferível a serem elicitadas, com I sendo o melhor valor e o pior valor é o. Atribui-se o valor de I para a primeira consequências e as demais recebem o valor de o. Comparando-se as consequências par a par começando com as consequências mais preferíveis, obtêm-se a relação de indiferença entre as duas consequências que pode ser expresso por $v\left(p_{I^{\prime}}, x_{2^{\prime}}, p_{3}, p_{4}\right)=v\left(m_{I^{\prime}} p_{2}, p_{3^{\prime}}, p_{4}\right)$, neste caso o valor da primeira constante será $k_{2} v_{2}=k_{I}$.

Etapa 5: Avaliação das outras constantes de escala

Repete-se o procedimento realizado na Etapa 4 para encontrar as demais constantes, mediante comparação par a par.

\section{Etapa 6: Finalização}

Realiza-se teste de consistência, por exemplo, sendo $x^{\prime}$ P (preferível a) $x^{\prime \prime}, \operatorname{logo} v\left(x^{\prime}\right)>$ $v\left(x^{\prime \prime}\right)$. Precisa-se nesse caso de $n-I$ equações, sendo $n$ o número de critérios.

\subsubsection{Etapa 9. Avaliação das Alternativas}

As alternativas são avaliadas conforme a problemática definida e uma visão geral é lançada sobre os resultados.

\subsubsection{Etapa 10. Análise de sensibilidade}

A análise de sensibilidade também é conhecida como análise de robustez, trata-se da análise de como variações em dados de entrada no modelo podem impactar os resultados da saída. Em uma problemática de ordenação como a apresentada no trabalho, por meio da análise de sensibilidade deseja-se saber o quanto modificações nos dados de entrada e parâmetros ou constantes podem impactar na alteração da ordem das alternativas.

Conforme Almeida (2013) existem duas formas ou dois tipos de análise de sensibilidade:

1) Para avaliação isolada de parâmetros ou tipo de dado de entrada

2) Para avaliação conjunta de todos os parâmetros e dados de entrada, ou um subconjunto com eles.

Para avaliação isolada de parâmetros ou tipo de dado de entrada tem-se que analisar o impacto da variação de um parâmetro ou dado de entrada, em particular, para o qual se decidiu simplificar sua obtenção durante o processo de modelagem (ALMEIDA, 20I3).

$\mathrm{Na}$ investigação do impacto da variação conjunta de dados e parâmetros utiliza-se o Método de Simulação conhecido como Monte Carlo. Neste procedimento simula-se a geração 
de números aleatórios para cada uma das entradas do modelo em conjunto, aplica-se e obtêm-se os resultados. A geração de números aleatórios deve compreender uma faixa de valores que tenha como base uma distribuição de probabilidade que represente fielmente os valores prováveis para os dados de entrada. Repete-se esse procedimento por meio do Método de Monte Carlo milhares de vezes e os resultados de saída são registrados para comparação com o resultado inicial obtido anteriormente, denominado resultado-padrão. Então, avalia-se o número de vezes que os resultados simulados diferem do resultado-padrão. Verificando-se o quanto o modelo é sensível a variações (ALMEIDA, 20I3).

\subsubsection{Etapa 11. Análise dos Resultados e Recomendações}

As alternativas analisadas serão confrontadas com incertezas e riscos associados aos dados de entrada, fraquezas presentes no processo decisório serão pontuadas e recomendações serão prescritas.

\subsubsection{Etapa 12. Implantação de Decisão}

Recomendações para a implementação dos resultados serão oferecidas ao decisor.

\section{Contextualização do Problema}

A organização alvo do estudo é um empreendimento de pequeno porte localizado em um município do interior do Pernambuco. A empresa processa dois tipos de frutas, a goiaba e a banana, para a produção de 6 produtos, a saber:
a) goiabada;
b) bananada;
c) goiabada em cubos;
d) bananada em cubos;
e) geléia de goiaba;
f) geléia de banana.

A confecção desses produtos na região e a implantação de empreendimentos desse tipo na cidade justificam-se pelo processamento da matéria-prima carecer de uma baixa quantidade de água; pela proximidade com centros produtores de frutas, como o Vale do Rio São Francisco, por exemplo; pela necessidade de mão-de-obra com baixa qualificação e pelo fato da região ter abundância de recursos energéticos, como lenha para o aquecimento de caldeiras.

Além disso, a cidade localiza-se na divisa com o estado da Paraíba e está próxima de importantes centros comerciais do Estado de Pernambuco. Levando em conta a alta concorrência que a empresa encontra no mercado de doces e a necessidade de especializar-se para cortar custos e oferecer aos clientes produtos com maior qualidade, a empresa pretende ordenar os seis produtos manufaturados pela companhia de forma que atenda aos objetivos estratégicos do decisor, sendo este, o dono e gerente da empresa. 


\section{Metodologia}

O presente trabalho pretende aplicar o Método de Agregação Aditivo Determinístico por meio do Modelo de Decisão Multicritério proposto por Almeida (2013) em uma problemática que busca a ordenação dos produtos manufaturados por uma empresa produtora de doces. Os produtos presentes no topo do ranking são preferíveis a serem manufaturados.

O modelo adotado foi discutido anteriormente e possui I2 etapas como mostra o Quadro I, todas as etapas estão interligadas e conectadas, sendo possível sempre que se queira voltar a etapa anterior para fazer as modificações que se julgar necessárias no processo.

Quadro I - Procedimento para resolução de problema de decisão

\begin{tabular}{|c|c|}
\hline ETAPA & MODELO DE DECISÃO MULTICRITÉRIO \\
\hline \multicolumn{2}{|c|}{ ANÁLISE PRELIMINAR } \\
\hline 1 & Carcterizar decisor(es) e outros atores \\
\hline 2 & Identificar objetivos \\
\hline 3 & Estabelecer critérios \\
\hline 4 & Estabelecer espaço de ação e problemática \\
\hline 5 & Identificar fatores não controlados \\
\hline \multicolumn{2}{|c|}{ FASE DE PREFERÊNCIAS E ESCOLHA DO MÉTODO } \\
\hline 6 & Efetuar modelagem de preferências \\
\hline 7 & Efetuar avaliação intracritério \\
\hline 8 & Efetuar avaliação intercritério \\
\hline \multicolumn{2}{|c|}{ FINALIZAÇÃO } \\
\hline 9 & Avaliar alternativas \\
\hline 10 & Efetuar análise de sensibilidade \\
\hline 11 & Analisar resultado e elaborar recomendações \\
\hline 12 & Implementar decisão \\
\hline
\end{tabular}

Fonte: adaptado Almeida (2013).

\section{Resultados}

Neste item do trabalho os resultados das I2 etapas do modelo são explicitados e discutidos.

\subsection{Resultado da Etapa 1. Caracterização dos Decisores e Outros Atores}

Em um primeiro instante cabe apresentar o organograma da empresa com os respectivos setores. A diretoria é composta pelo dono da empresa que também é diretor e administrador do empreendimento, o Setor Financeiro é composto por um auxiliar financeiro responsável pela contabilidade da empresa, o setor de Recursos Humanos trata de assuntos ligados ao pessoal e o Setor Operacional é liderado por um supervisor sob a responsabilidade do qual encontra-se os colaboradores do "chão-de-fábrica".

Nesse caso, o decisor será o dono da empresa. O analista será um indivíduo externa a organização que captará informações junto aos demais setores do empreendimento para o estabelecimento dos critérios e alternativas e a confecção do modelo. O especialista também será um indivíduo externo a organização que fornecerá informações especializadas, como por 
exemplo, a mensuração do grau de aceitabilidade dos produtos no mercado, para tanto é preciso de conhecimento especializado. Os stakeholders neste caso são os consumidores e fornecedores que sofrerão as consequências tomadas pelo decisor.

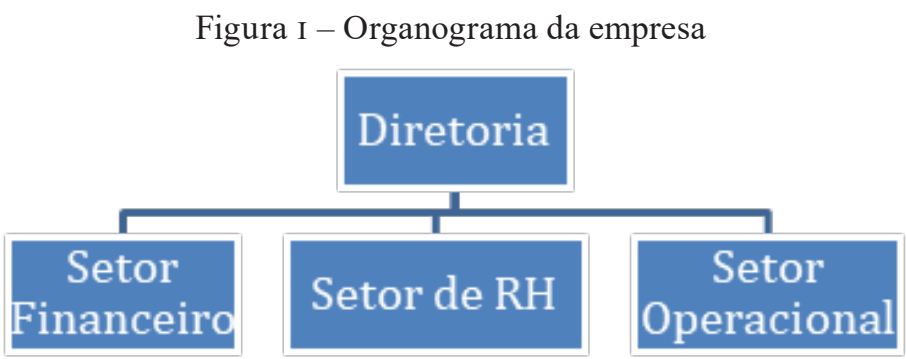

Fonte: Autoria Própria (2019).

\subsection{Resultado da Etapa 2. Identificação dos Objetivos}

Para o problema, o objetivo fundamental seria ordenar e escolher entre os doces processados no estabelecimento os produtos que possuem menor custo de produção, e maior rendimento e qualidade percebida entre consumidores. Logo os doces seriam configurados como alternativas para alcançar aos objetivos.

Pra atingir o objetivo fim, os objetivos - meio seriam:

a) Redução do tempo de preparo de cada quilograma do produto em horas;

b) Redução do preço da embalagem;

c) Aumento do rendimento de produto por quilograma de polpa processada;

d) Redução do custo de produção de cada quilograma do produto processado;

e) Redução do peso líquido de cada produto embalado;

f) Maximização da aceitabilidade ou aceitação do produto no mercado.

\subsection{Resultado da Etapa 3. Estabelecimento dos Critérios}

Para a avaliação das alternativas do problema foram determinados seis critérios, julgados pelo decisor, com o auxílio do especialista, como importantes para a análise do problema em questão. Cabe salientar que os critérios são medidas de desempenho ligadas aos objetivos. Dessa forma os critérios são elencados abaixo:

a) $\mathrm{O}$ tempo de preparo de cada quilograma do produto em horas: quanto menor, mais preferível a alternativa. O tempo de preparo foi calculado tendo como base os processos e a rotina de trabalho consolidado da instituição, visto que existe padronização nos tempos de processamento, menor tempo de preparo pode em alguns casos significar menos mão-de-obra necessária na produção e/ou menos ociosidade no trabalho.

b) Preço da Embalagem: quanto menor, mais preferível a alternativa. Embalagem mais baratas significam a possibilidade de aumentar a margem de lucro, visto que o custo da embalagem impacta no preço final dos produtos produzidos. 
c) Rendimento de produto por quilograma de polpa processada: quanto maior, melhor a alternativa. Durante o cozimento as polpas processadas perdem água e necessitam de quantidades diferentes de açúcar ou outros ingredientes para alcançar o "ponto" do doce. Dessa forma, os doces processadas possuem rendimentos diferentes quando se considera a mesma quantidade de polpa acrescentada para cada produto e seus respectivos resultados finais. Como a receita é padronizada tem-se o rendimento final de cada produto por quilograma utilizado na receita inicialmente.

d) Custo de produção de cada quilograma do produto processado: quanto menor, melhor a alternativa. Corresponde o custo de materiais necessários para se produzir cada quilo de receita de cada doce, além da mão-de-obra e custos fixos associados.

e) Peso líquido de cada produto embalado: quanto menor, melhor a alternativa. Produtos mais elaborados, melhor acabados, com alto valor agregado e com baixo peso para comercialização são mais lucrativos para a empresa.

f) Aceitabilidade mo mercado: quanto maior menor, melhor a alternativa. Produtos que possuem melhor aceitação entre os consumidores, consequentemente são os que mais são vendidos. Os valores de aceitabilidade ou aceitação correspondem à média das notas que consumidores atribuíram aos diversos produtos/alternativas por meio de uma pesquisa de mercado. Durante a pesquisa pediu-se que os consumidores avaliassem a qualidade dos produtos em uma escala entre 0 e 100, para tanto foi utilizada uma amostra com 60 consumidores.

Quanto à escala de avaliação, o critério tempo de preparo pode ser classificado como intervalar, os demais critérios: preço da embalagem, rendimento por quilograma de polpa processada, custo do quilograma de polpa processada, peso por embalagem do produto por embalagem processada e aceitabilidade no mercado podem ser classificados como de razão.

\subsection{Resultado da Etapa 4. Estabelecimento do Espaço de Ações e Problemática}

Abaixo, pode-se visualizar a matriz de conseqüência, Tabela I, dos critérios selecionados para o problema e seus respectivos valores. As alternativas são os produtos manufaturados pela empresa:

Tabela I - Matriz consequências do problema

\begin{tabular}{|c|c|c|c|c|c|c|}
\hline & $\begin{array}{c}\text { Tempo de } \\
\text { preparo } \\
\text { Kg/Hrs } \\
\text { (horas) }\end{array}$ & $\begin{array}{c}\text { Embalagem } \\
(\$)\end{array}$ & $\begin{array}{c}\text { Rendimento } \\
\text { por Kg de } \\
\text { polpa (taxa) }\end{array}$ & $\begin{array}{c}\text { Custo } \\
\text { por Kg } \\
\text { produzido } \\
(\$)\end{array}$ & $\begin{array}{c}\text { Peso por } \\
\text { produto } \\
\text { embalado em } \\
\text { Kg (peso) }\end{array}$ & $\begin{array}{c}\text { Aceitabilidade } \\
\text { no mercado } \\
\text { (taxa) }\end{array}$ \\
\hline Goiabada & 0,25 & 0,10 & 1,375 & 4,00 & 0,300 & 0,6 \\
\hline $\begin{array}{c}\text { Goiabada } \\
\text { em Cubos }\end{array}$ & 0,42 & 0,50 & 1,438 & 4,30 & 0,350 & 0,75 \\
\hline $\begin{array}{c}\text { Geléia de } \\
\text { Goiaba }\end{array}$ & 0,15 & 2,50 & 1,250 & 3,60 & 0,200 & 0,65 \\
\hline Bananada & 0,42 & 0,10 & 0,890 & 3,70 & 0,300 & 0,8 \\
\hline $\begin{array}{c}\text { Bananada } \\
\text { em Cubos }\end{array}$ & 0,50 & 0,50 & 0,970 & 4,00 & 0,350 & 0,6 \\
\hline $\begin{array}{c}\text { Geléia de } \\
\text { Banana }\end{array}$ & 0,25 & 2,50 & 0,830 & 3,40 & 0,200 & 0,8 \\
\hline
\end{tabular}

Fonte: Autoria Própria (20I9). 
O presente problema pode ser classificado como uma Problemática de Ordenação (P. $\gamma)$, pois quer-se ordenar as melhores alternativas dentre os produtos confeccionados pelo empreendimento.

\subsection{Resultado da Etapa 5. Identificação de Fatores Não Controlados}

Fatores não controlados são fatores não explícitos ou sujeitos a incertezas e risco. Apesar de existirem intervalos padronizados para o tempo de processamento de cada polpa de fruta, assim como o rendimento médio para cada quilograma de polpa ser conhecido, variações no rendimento podem ocorrer caso durante o processamento o doce 'passe do ponto', ou algum funcionário fique doente e atrase o processo, por exemplo. A aceitabilidade, ou aceitação do mercado, pode não corresponder à realidade devido à pequena amostra de consumidores, ou as respostas podem ser enviesadas por determinados fatores da pesquisa.

\subsection{Resultado da Etapa 6. Modelagem de Preferências}

Por meio da análise do problema verificou-se que a estrutura que mais se adequa as preferências dos decisores para o problema é a estrutura (P,I), uma vez que foi possível comparar todas as alternativas por meio das relações de preferência estrita $(\mathrm{P})$ ou indiferença (I) (comprovando-se a ordenabilidade), obtendo-se uma relação simétrica e outra assimétrica. Posteriormente, observou-se que há por parte do decisor a propriedade de transitividade. Dessa forma, quando essas duas propriedades são atendidas o método recomendado para o problema é o de critério único de síntese (modelo de agregação aditivo). A racionalidade do decisor para o problema foi identificada como compensatória, ou seja, existe a ideia de compensar um menor desempenho de um critério em uma alternativa por outro critério.

\subsection{Resultado da Etapa 7. Avaliação Intracritério}

A Tabela 2 exibe os dados do problema normalizados utilizando-se as Equações I e 2. A normalização dos critérios de Tempo de Preparo, Embalagem, Custo por Quilograma Produzido e Peso por Produto Embalado em Quilograma ocorreu conforme a Equação 5, pois são objetivos que deseja-se minimizar, os demais critérios por meio da Equação 4.

Tabela 2 - Matriz decisão do problema

\begin{tabular}{|c|c|c|c|c|c|c|}
\hline & $\begin{array}{c}\text { Tempo de } \\
\text { preparo } \\
\text { Kg/Hrs } \\
\text { (horas) }\end{array}$ & $\begin{array}{c}\text { Embalagem } \\
(\$)\end{array}$ & $\begin{array}{c}\text { Rendimento } \\
\text { por Kg de } \\
\text { Polpa (taxa) }\end{array}$ & $\begin{array}{c}\text { Custo } \\
\text { por Kg } \\
\text { Produzido } \\
(\$)\end{array}$ & $\begin{array}{c}\text { Peso por } \\
\text { produto } \\
\text { embalado em } \\
\text { Kg (peso) }\end{array}$ & $\begin{array}{c}\text { Aceitabilidade } \\
\text { no Mercado } \\
\text { (taxa) }\end{array}$ \\
\hline Goiabada & 4,00000 & 10,0000 & 0,89638 & 0,25000 & 3,33333 & 0,00000 \\
\hline $\begin{array}{c}\text { Goiabada } \\
\text { em Cubos }\end{array}$ & 2,38095 & 2,0000 & 1,00000 & 0,23256 & 2,85714 & 0,75000 \\
\hline $\begin{array}{c}\text { Geléia de } \\
\text { Goiaba }\end{array}$ & 6,66667 & 0,4000 & 0,69079 & 0,27778 & 5,00000 & 0,25000 \\
\hline Bananada & 2,38095 & 10,0000 & 0,09868 & 0,27027 & 3,33333 & 1,00000 \\
\hline $\begin{array}{c}\text { Bananada } \\
\text { em Cubos }\end{array}$ & 2,00000 & 2,0000 & 0,23026 & 0,25000 & 2,85714 & 0,00000 \\
\hline $\begin{array}{c}\text { Geléia de } \\
\text { Banana }\end{array}$ & 4,00000 & 0,4000 & 0,00000 & 0,29412 & 5,00000 & 1,00000 \\
\hline
\end{tabular}

Fonte: Autoria Própria (20I9). 


\subsection{Resultado da Etapa 8. Avaliação Intercritério}

Esta fase marca a interferência do decisor no processo metodologico, pois os pesos associados a cada critério irão representar o quanto o decisor compensa a consequência de um critério em relação a outro.

Neste trabalho para a elicitação das constantes de escala utilizou-se um software do Centro de Desenvolvimento em Sistemas de Informação e Decião (CDSID) que realizou o trade-off entre o critérios estabelecidos, gerando portanto a Tabela 3. Nesta Tabela pode-se observar a referência do critério, classificados de i a 6, a descrição e o valor das respectivas constantes ou taxa de substituição, que somam I na totalidade.

Tabela 3 - Valor de elicitação das constantes de escala

\begin{tabular}{|c|c|c|}
\hline Critério & Descrição & Valor da Constante \\
\hline 1 & Tempo de preparo Kg/Hrs (horas) & 0,00993 \\
\hline 2 & Embalagem $(\$)$ & 0,02271 \\
\hline 3 & Rendimento por Kg de polpa (taxa) & 0,18164 \\
\hline 4 & Custo por Kg produzido (\$) & 0,77501 \\
\hline 5 & Peso por produto embalado em Kg (peso) & 0,00326 \\
\hline 6 & Aceitabilidade no mercado (taxa) & 0,00745 \\
\hline
\end{tabular}

Fonte: Autoria Própria (2019).

\subsection{Resultado da Etapa 9. Avaliação das Alternativas}

Após a obtenção das constantes de escala, tem-se efetivamente a aplicação do método multicritério para a problemática de ordenação. A Tabela 4 contém o resultado final com a agregação das preferências do decisor, após a multiplicação da constante pelo valor normalizado para cada critério (Tabela 2).

Tabela 4 - Matriz decisão do problema com normalização e constantes de escala

\begin{tabular}{|c|c|c|c|c|c|c|c|c|}
\hline & Critério 1 & Critério 2 & Critério 3 & Critério 4 & Critério 5 & Critério 6 & $\begin{array}{c}\text { Valor } \\
\text { Global }\end{array}$ & Ordem \\
\hline $\mathrm{k}_{\mathrm{j}}$ & 0,00993 & 0,02271 & 0,18164 & 0,77501 & 0,00326 & 0,00745 & - & - \\
\hline Goiabada & 4,00000 & 10,0000 & 0,89638 & 0,25000 & 3,33333 & 0,00000 & 0,63426 & 1 \\
\hline $\begin{array}{c}\text { Goiabada } \\
\text { em Cubos }\end{array}$ & 2,38095 & 2,0000 & 1,00000 & 0,23256 & 2,85714 & 0,75000 & 0,44584 & 3 \\
\hline $\begin{array}{c}\text { Geléia de } \\
\text { Goiaba }\end{array}$ & 6,66667 & 0,4000 & 0,69079 & 0,27778 & 5,00000 & 0,25000 & 0,43420 & 4 \\
\hline Bananada & 2,38095 & 10,0000 & 0,09868 & 0,27027 & 3,33333 & 1,00000 & 0,49645 & 2 \\
\hline $\begin{array}{c}\text { Bananada } \\
\text { em Cubos }\end{array}$ & 2,00000 & 2,0000 & 0,23026 & 0,25000 & 2,85714 & 0,00000 & 0,31017 & 5 \\
\hline $\begin{array}{c}\text { Geléia de } \\
\text { Banana }\end{array}$ & 4,00000 & 0,4000 & 0,00000 & 0,29412 & 5,00000 & 1,00000 & 0,30050 & 6 \\
\hline
\end{tabular}

Fonte: Autoria Própria (20I9). 


\subsection{Etapa 10. Análise de Sensibilidade}

Para o presente trabalho utilizou-se a análise de sensibilidade que varia o valor das constantes associadas aos critérios.

Na primeira interação, o valor da constante $\mathrm{k}_{5}$ foi aumentado em $20 \%$, esse valor foi imediatamente subtraído do valor da constante $\mathrm{k}_{4}$, como resultado tem-se a Tabela 5 .

Tabela 5 - Análise de sensibilidade I

\begin{tabular}{|c|c|c|c|c|c|c|c|c|}
\hline & Critério 1 & Critério 2 & Critério 3 & Critério 4 & Critério 5 & Critério 6 & $\begin{array}{c}\text { Valor } \\
\text { Global }\end{array}$ & Ordem \\
\hline $\mathrm{k}_{\mathrm{i}}$ & 0,00993 & 0,02271 & 0,18164 & 0,771098 & 0,003912 & 0,00745 & - & - \\
\hline Goiabada & 4,00000 & 10,0000 & 0,89638 & 0,25000 & 3,33333 & 0,00000 & 0,63545 & 1 \\
\hline $\begin{array}{c}\text { Goiabada } \\
\text { em Cubos }\end{array}$ & 2,38095 & 2,0000 & 1,00000 & 0,23256 & 2,85714 & 0,75000 & 0,44679 & 3 \\
\hline $\begin{array}{c}\text { Geléia de } \\
\text { Goiaba }\end{array}$ & 6,66667 & 0,4000 & 0,69079 & 0,27778 & 5,00000 & 0,25000 & 0,43638 & 4 \\
\hline Bananada & 2,38095 & 10,0000 & 0,09868 & 0,27027 & 3,33333 & 1,00000 & 0,49756 & 2 \\
\hline $\begin{array}{c}\text { Bananada } \\
\text { em Cubos }\end{array}$ & 2,00000 & 2,0000 & 0,23026 & 0,25000 & 2,85714 & 0,00000 & 0,31106 & 5 \\
\hline $\begin{array}{c}\text { Geléia de } \\
\text { Banana }\end{array}$ & 4,00000 & 0,4000 & 0,00000 & 0,29412 & 5,00000 & 1,00000 & 0,30261 & 6 \\
\hline
\end{tabular}

Fonte: Autoria Própria (20I9).

Em uma segunda interação variou-se o valor da constante $\mathrm{k}_{5}$ aumentado em $20 \%$, esse valor foi então subtraído do valor da constante $\mathrm{k}_{3,}$ como mostra a Tabela 6 .

Tabela 6 - Análise de sensibilidade 2

\begin{tabular}{|c|c|c|c|c|c|c|c|c|}
\hline & Critério 1 & Critério 2 & Critério 3 & Critério 4 & Critério 5 & Critério 6 & $\begin{array}{c}\text { Valor } \\
\text { Global }\end{array}$ & Ordem \\
\hline $\mathrm{k}_{\mathrm{j}}$ & 0,00993 & 0,02271 & 0,18164 & 0,771098 & 0,003912 & 0,00745 & - & - \\
\hline Goiabada & 4,00000 & 10,0000 & 0,89638 & 0,25000 & 3,33333 & 0,00000 & 0,63643 & 1 \\
\hline $\begin{array}{c}\text { Goiabada } \\
\text { em Cubos }\end{array}$ & 2,38095 & 2,0000 & 1,00000 & 0,23256 & 2,85714 & 0,75000 & 0,44770 & 3 \\
\hline $\begin{array}{c}\text { Geléia de } \\
\text { Goiaba }\end{array}$ & 6,66667 & 0,4000 & 0,69079 & 0,27778 & 5,00000 & 0,25000 & 0,43746 & 4 \\
\hline Bananada & 2,38095 & 10,0000 & 0,09868 & 0,27027 & 3,33333 & 1,00000 & 0,49862 & 2 \\
\hline $\begin{array}{c}\text { Bananada } \\
\text { em Cubos }\end{array}$ & 2,00000 & 2,0000 & 0,23026 & 0,25000 & 2,85714 & 0,00000 & 0,31203 & 5 \\
\hline $\begin{array}{c}\text { Geléia de } \\
\text { Banana }\end{array}$ & 4,00000 & 0,4000 & 0,00000 & 0,29412 & 5,00000 & 1,00000 & 0,30376 & 6 \\
\hline
\end{tabular}

Fonte: Autoria Própria (20I9).

Como mostra as Tabelas 5 e 6, a ordenação das alternativas não foi modificada com a adição das mudanças feitas nos valores das constantes, dessa forma exemplificou-se e verifica-se a sensibilidade do modelo a variações nos valores das constantes. Cabe salientar que esse é apenas um exemplo e milhares de interações que devem ou podem ser realizadas para comprovar a robustez do modelo. 


\subsection{Etapa 11. Análise dos Resultados e Recomendações}

Conforme pode-se observar na Tabela 4 as melhores alternativas para escolha de produção são goiabada, bananada, goiabada em cubos, geléia de goiaba, bananada em cubos e geléia de banana respectivamente. Também cabe relatar as incertezas e riscos associados aos dados de entrada e parâmetros e seu impacto no modelo, todos esses detalhes devem ser apresentados ao decisor de forma clara e concisa.

Neste trabalho tiveram-se incertezas e riscos ligados ao estabelecimento do tempo médio de preparo de cada doce, visto que existem variações na programação de produção dos processos produtivos, alguns imprevistos como atrasos e diminuição do ritmo de trabalho podem ocorrer.

Para a análise da aceitação dos produtos, incertezas podem surgir no estabelecimento da escala de valor de avaliação da qualidade dos produtos produzidos ou a própria pesquisa pode ser enviesada pelo aplicador.

\subsection{Etapa 12. Implantação de Decisão}

Após a ordenação das melhores alternativas baseadas na preferência do decisor, deve-se estabelecer um prazo para a implantação das ações de forma que o decisor tenha tempo de pensar e amadurecer as ideias e alternativas obtidas no modelo.

\section{Conclusão}

O presente trabalho aplicou o Método de Agregação Aditivo Determinístico para a ordenação da melhor alternativa de produto a ser confeccionado por uma fábrica de doces no Estado de Pernambuco, obtiveram-se os resultados, realizou-se a análise de sensibilidade e foram apontadas as fraquezas presentes no processo de implementação do modelo.

O modelo aplicado caracteriza-se por possuir uma abordagem construtivista e buscar captar as preferências do decisor, fato observado na elicitação da constante de escala.

Cabe salientar que o Modelo de Tomada de Decisão apresentado por Almeida (20I3) pode ser utilizado para a aplicação de diferentes métodos multicritério, nesse caso, foi utilizado o Método de Agregação Aditivo Determinístico, cuja aplicação ocorreu de maneira mais didática, se este trabalho for comparado com as publicações presentes no breve estudo do estado da arte.Logo, a forma como o Método foi aplicado ao Modelo corroboraria para a discussão e aplicação desse mesmo Modelo com outros métodos.

O estudo do estado da arte, por sua vez, oferece uma rápida visão geral sobre as publicações que consideram o Método Multicritério Aditivo em português, onde verificou-se o parco número de trabalhos sobre o assunto mediante os critérios estabelecidos para a pesquisa.

Como limitações do trabalho pode-se destacar que durante o processo decisório devido à racionalidade limitada do decisor alguns erros podem ter ocorrido na elicitação das constantes ou em processos que requeiram grande esforço cognitivo. 
Incertezas podem surgir durante a definição do tempo médio de processamento de cada doce e da definição do rendimento da polpa. Erros de escala e viés na verificação da aceitação dos produtos junto ao mercado também podem ocorrer e influenciar na qualidade da resposta obtida.

Para trabalhos futuros propõe-se a aplicação de outros Métodos Aditivos, ou Métodos Aditivos com veto, para ordenação das alternativas do problema e comparação com os resultados obtidos nesse trabalho, bem como destaca-se a possibilidade de utilizar o FITradeoff ,conforme sugere Almeida et al. (20I6), na elicitação da constante de escala em contraponto ao Tradeoff tradicional utilizado nesse trabalho.

Também seria conveniente para trabalhos futuros o estudo do estado da arte do comportamento dos vetos em métodos multicritério compensatórios. Bem como uma pesquisa aprofundada do estado da arte referente ao Método de Agregação Aditivo Determinístico e ao Método de Agregação Aditivo Determinístico com Veto para embasar trabalhos futuros.

\section{REFERÊNCIAS}

ALMEIDA, A. T. Processo de Decisão nas Organizações: Construindo Modelos de decisão multicritério. São Paulo: Atlas, 2013.

ALMEIDA, A. T.; ALMEIDA, J. A.; COSTA, A. P. C. S.; ALMEIDA FILHO, A. T. A new method for elicitation of criteria weights in additive models: Flexible and interactive tradeoff. European Journal of Operational Research, v. 250, p. I79-I9I, 2016.

BOUYSSOU, D.; MARCHANT, T.; PIRLOT, M.; TSOUKIAS, A.; VINCKE, P. Evaluation and decision models with multiple criteria. Springer, 2006.

DAHER, S. S. D.; ALMEIDA, A. T. The use of ranking veto concept to mitigate the compensatory effects of additive aggregation in group decisions on a water utility automation investment. Group Decision and Negotiation, v. 2I, n. 2, p. I85-204, $20 \mathrm{I} 2$.

DE ALMEIDA, A. T. Additive-veto models for choice and ranking multicriteria decision problems. Journal of Operational Research, v.30, n.6, p.I-20, 2013.

EDWARDS, W.; MILES JUNIOR, R. F.; WINTERFELDT, D. Advances in decision analysis: from foundations to applications. Cambridge : Cambridge University Press, 2007.

EISENHART, K. M.; ZBARACKI, M. J. Strategic Decision Making. Strategic Management Journal, v.I3, p. I7-37, 1992.

EVAIR, J.; AUGUSTO, H.; LUIZA, M.; VALERIANO, M.; TURET, J. Aplicação do modelo aditivo com veto para a problemática de escolha de um novo fornecedor: estudo de caso. In: Congresso Brasileiro de Engenharia de Produção. 8., 20I8, Ponta Grossa. Anais [...]. Ponta Grossa: APREPRO, 20I8. p. I-I2. 
GOLDBARG, M. C., GOLDBARG, H., LUNA, P. L. Otimização combinatória e programação linear: modelos e algoritmos. 2. ed. Rio de Janeiro: Elsevier, 2005.

HILLIER, F., LIEBERMAN, G. Introduction to operations research. New York: McGrawHill Education, 2015.

JANIS, I. L. Crucial decissions. New York: Free Press, 1989

KEENEY, R. L. Value focused thinking. Harvard: Harvard University Press, 1992.

LACHTERMACHER, G. Pesquisa operacional na tomada de decisões: modelagem em Excel. Rio de Janeiro: Elsevier, 2007.

PARDALOS, P. M.; SISKOS, Y.; ZOPOUNIDIS, C. Advances in multicriteria analysis. New York: Kluwer Academic Publishers, 1995.

PINTO, K. C. R. Aprendendo a decidir com a pesquisa operacional: modelos e métodos de apoio a decisão. 2 ed. Urbelândia: UFU, 2006.

PINTO JUNIOR, R. P. S.; MELLO, J. C. C. B. S. Identificação da melhor escolha de funcionário para realização de inspeção em estatais do setor elétrico. Prodiction. São Paulo, v. 23, n.I, p. I35 - I43, $20 \mathrm{I} 3$

ROY, B. Multicriteria methodology goes decision aiding. New York: Kluwer Academic Publishers. 1996.

SANTOS, L. V. B.; CASADO, R. S. G. R.; CARVALHO, E. N.; SILVA, L. C. Aplicação do método aditivo com veto no processo de priorização de fornecedores: estudo de caso em uma empresa de confecções. In: Simpósio Brasileiro de Pesquisa Operacional, 49., 20I7, Blumenau. Anais [...]. Blumenau: SOBRAPO, 20I7. p. I - I2.

SANTOS, V. A. P.; LUGO, S. D. R. ALMEIDA, A. T. Decisão multicritério por critério único de síntese: aplicação a uma compra de tecnologia. In: Encontro Nacional de Engenharia de Produção. 35., 20I5, Fortaleza. Anais [...]. Fortaleza: ABEPRO, 20I5. p. I- 24.

SAYÃO, L. F. Métodos teóricos em ciência da informação: abstração e método científico

Ciência da Informação, Brasília, v. 30, n. I, p. 82-9I, jan./abr. $200 I$.

SEBRAE, Serviço Brasileiro de Apoio a Micro e Pequenas Empresas. Micro e Pequenas Empresas na Economia Brasileira e de Pernambuco, 20I7. Disponível em: $\quad$ http//:oemmndcbldboiebfnladdacbdfmadadm/http://www.pe.agenciasebrae.com.br/ asn/Estados/PE/Indicadores/PERNAMBUCO\%2oindicadores\%20de\%20MPE_20I6_ atualizado\%20em\%20I0maioI7.pdf. Acesso em: OI abr. 2020.

SHARDA, R.; DELEN, D.; TURBAN, E. Business intelligence and analytics: systems for 
decision support . IO ed. Pearson, 2015.

SIMON, H. Administração behavior. 4 ed. New York: The Free Press, 1997.

SLACK, N.; JONES, A. B.; JOHNSTON, R. Administração da Produção. 4. ed. São Paulo: Atlas, 2016.

TURET, J. G.; DAHER, S. F. D. Aplicação de modelo multicritério para elaboração de plano de ação em m-commerce. In: Simpósio Brasileiro de Pesquisa Operacional. 47., 20I5, Porto de Galinhas. Anais [...]. Porto de Galinhas: SOBRAPO, 20I5. p. $232-243$.

VINCKE, P. Multicriteria decision-aid. Bruxelles: John Wiley \& Sons, 1992.

WALLENIUS, J.; DYER, J. S.; FISHBURN, P. C.; RALPH E. STEUER, R. E.; ZIONTS, S.; $\underline{\mathrm{DEB}}, \mathrm{K}$. Multiple criteria decision making, multiattribute utility theory: recent accomplishments and what lies ahead. Management science. v. 54. , n. 7, p. I336-I349, 2008. 